\title{
Simulation tool for dimensioning power train of hybrid working machine
}

\author{
Anna Tupitsina ${ }^{1}$ Jan-Henri Montonen ${ }^{1}$ Jani Alho $^{1}$ Paula Immonen ${ }^{2}$ \\ Mika Lauren ${ }^{3}$ Pia Lindh ${ }^{1}$ Tuomo Lindh ${ }^{1}$ \\ ${ }^{1}$ Department of Electrical Engineering, Lappeenranta-Lahti University of Technology LUT, Finland. E-mail: \\ \{Anna.Tupitsina, Henri.Montonen, Jani.Alho, Pia.Lindh, Tuomo.Lindh\} @lut.fi \\ ${ }^{2}$ LES Common Activities, Lappeenranta-Lahti University of Technology LUT, Finland. E-mail: \\ Paula.Immonen@lut.fi \\ ${ }^{3}$ Department of Mechanical Engineering, Turku University of Applied Sciences, Finland. E-mail: \\ Mika.Lauren@turkuamk.fi
}

\begin{abstract}
The tightening of emission standards and related regulations leads to the necessity of the hybridization of mobile machines. The working operation profile of non-road machinery differs for diverse application types and often varies on large scale. Since the series production of mobile vehicles is generally relatively limited, their design can be tailored to a specific driving cycle. Therefore, this work aims to introduce a simulation tool, which is flexible for the initial design of a non-road hybrid electric vehicle (HEV) drivetrain when different architectures (series and parallel) and dimension of components are considered. The dimensioning is based on the load cycles, that describe the power of the machine during the operational process. For the ease of the first design, each component is modeled mainly using the data available from manufacturers. Case studies are provided to illustrate the use of the simulation tool, where different options of the dimensioned hybrid powertrain are considered.
\end{abstract}

Keywords: Hybrid working machine, power train, simulation tool

\section{Introduction}

The tightening of emission standards and related regulations (ICCT, 2016) leads to the necessity of improvement of a conventional mobile machine and attracting attention to the hybridization of vehicles. Hybrid electrical vehicles (HEV) provide a great potential to reduce fuel consumption (FC) and diminish exhaust gas emissions since the workload of a machine is distributed between an internal combustion engine (ICE) and energy storage (ES). It allows to reduce the size of a diesel engine and enables its operation in the most efficient operating modes. Likewise, HEV technology facilitates vehicle performance improvements, such as energy efficiency and controllability (Lajunen et al., 2018), as well as provides flexibility in the layout design of a vehicle (Moreda et al., 2016).

Hybridization is gaining popularity for off-road machinery (Wang et al., 2016), (He and Jiang, 2018) since great performance and consumption improvements can be achieved for a hybrid vehicle with widely varying load power (Lajunen et al., 2018). Most recent articles and studies have described case examples of mobile machine hybridization (Mocera and Som, 2020) (Ishida and Higurashi, 2015), where models of HEV components are produced for the specified system under consideration with the predetermined technical characteristics. Since the series production of non-road vehicles is generally relatively limited, their design can be tailored to a specific driving cycle (Lajunen et al., 2018). 
There is no common general drive cycle, but the working operation profile of mobile machines often varies in a large scale (Lajunen et al., 2016), (EPA, 2021). Furthermore, different HEV topologies options can be considered for a particular work cycle. Topologies are classified according to the traction system type: series and parallel (Enang and Bannister, 2017). The parallel system offers the slightest changes to the conventional topology, where an ICE and an electrical machine (EM) are connected mechanically to the transmission. In the series hybrid, there is no mechanical connection between an ICE and a traction drive, the machine is propelled electrically.

Different topology options require different dimensions of components. The sizing of HEV components is complicated (Huang et al., 2018) by the fact that there are different energy sources with their auxiliary devices and multiple power flow paths with different architectures. Moreover, the dimensioning of various components specifies the limits of operation of each element and defines their dynamics and efficiency. Those aspects complicate the evaluation of the performance of the vehicle, FC, and the feasibility of different control strategies.

Diverse variation in work cycles of non-road vehicles with multiple hybridizations and dimension choices create a demand for a calculation schema to determine the feasible architecture and sufficient component size of HEV for a given duty cycle. Therefore, the following aspects should be considered in the calculation schema: a) execution of the known work cycles (torque and velocity curves are specified), b) changing the topology of a vehicle, and c) testing different diesel engines, ES, and electrical drives. However, direct scaling cannot be implemented for some components due to different sizes and a variety of types. For example, only a few suitable combustion engines may be available, or in the case of electrical motors, efficiency maps vary between motor size, efficiency class, and, especially, motor type. It causes an additional precondition to the simulation tool: the model of each component should be based on the available data.

Different simulation packages to model HEV have been introduced over the past years. Those tools are based on MATLAB/Simulink and Simscape (ADVISOR, PSAT), on Modelica language, etc. (Tammi et al., 2018). However, most of the simulation platforms require detailed models based on measured experimental data (Gao et al., 2007) (Markel et al., 2002) (Evangelou and Shabbir, 2016), which complicates the task of studying different hybridization options, if components with required dimensions are not available in the database. A scalable engine model was presented in (Rizzoni et al., 1999). However, ex- perimental data measurements are needed to fit unknown coefficients to obtain parametrization equations describing ICE with demanded size (Pisu and Rizzoni, 2007). A simulation tool that does not rely on empirical data would provide an opportunity to do initial estimation without additional expenses on gaining those data.

The aim of this work is to introduce a simulation tool, which is relatively flexible for the initial design of non-road mobile HEVs when different architectures and component dimensions are considered. The exact simulation of each component of a $\mathrm{HEV}$ is not of high priority, but models should provide comparable results of energy efficiency, and FC, and performance of different hybrid systems and dimensions. With widely varying operation modes, the HEV system should further capture the main dynamics to sufficiently evaluate the FC and performance of a vehicle. When a hybrid powertrain is dimensioned, not only the question of minimized FC is considered, but also the layout of components within available space and topology selection. However, this question is out of the scope of this research. In addition, the temperature effect due to power losses of the components is not taken into account since the cooling agents and mechanism varies between manufacturers, and modeling requires the data which are not typically provided in data sheets. For the initial estimation, if overloading occurs, the acceptable duration and value should not exceed the performance characteristics determined by a manufacturer.

The simulation models describe the power flow in a hybrid system, in which all components are modeled in a modular way for the convenience of single element modification and topology changes. Parameters and dimensions of each element can be changed easily, according to the accessible data from manufacturers. The dimensioning is based on a load cycle, which can be standardized or recorded by a manufacturer data. Virtual testing of a mobile machine can contribute to the evaluation of $\mathrm{HEV}$ dimensions since detailed information about energy flows in the system can be obtained. Real-time Multi-Body Dynamics (MBD) simulation was used to compare FC and productivity of the series hybrid diesel-electric versions of a mine loader (Nokka et al., 2017). The simulation was carried out as co-simulation, in which the powertrain was modeled in Simulink and the rest of the machine and the environment in the MBD simulator. This study builds upon previous research (Immonen, 2013). However, modifications have been made regarding the ICE model, ES, and control strategy of series and parallel hybrid, as well as the use of load-cycle data in simulation. 


\section{Simulation model description}

The proposed simulation tool enables dimensioning of each element according to the off-road machine power and type and modify system layout in line with the hybridization topology. The simulation is based on the idea of power flows and power balance calculations. The parallel and series topologies are obtained by different connections of drivetrain components. In a typical parallel hybrid topology Figure 1, an ICE axle and an electrical drive axle (ED in the figure) are directly connected to the torque coupler, where the main purpose of the controller is to determine the torque value simulated by each motor. The reference values to the blocks are annotated with ref, while the simulated outputs are presented without it. The sign of reference torque value of the electrical drive determines the direction of the applied torque. If the value is positive then the power is transferred from ES to electrical drive discharging the ES. If negative, the direction is reversed, and ES is charging. The available capacity is represented by the State of the Charge (SOC). The simulated torque from the electrical drive and the ICE is transferred to the torque coupler that drives the vehicle. The power flow through each component, as well as the power balance of the system, are calculated through modeled torque and velocity. In the exemplary series hybrid Figure 2, the diesel engine is decoupled from the mechanical transmission and connected to the electrical drive via the generator and the $\mathrm{DC}$ bus. If the power from the diesel engine is insufficient to power the vehicle, the ES serves as a second power source. Otherwise, it stores the excessive power of the hybrid system. The controller determines the required torque for all machines in regard to the ES SOC and load profile. In the simulation, the vehicle model is replaced by standardized or recorded load cycles, where the velocity of a vehicle is determined and supplied to wheels. Alternatively, the vehicle model can be simulated in an MBD simulator.

Essential HEV units to be designed are an ICE, an electrical drive, ES, and a transmission system. Some models of HEV components are purely static, some are quasi-static, meaning that the operation point is changing considering the main dynamics (lags) behavior. All component models include loss calculation. The use of loading curves is straightforward but requires that mechanical components are modeled as rigid bodies. Though, with suitable loading calculations and control, flexible bodies could be included. This kind of loading model is presented in (Immonen, 2013). For initial dimensioning and energy management control, the dynamic behavior of flexible bodies is not essential.

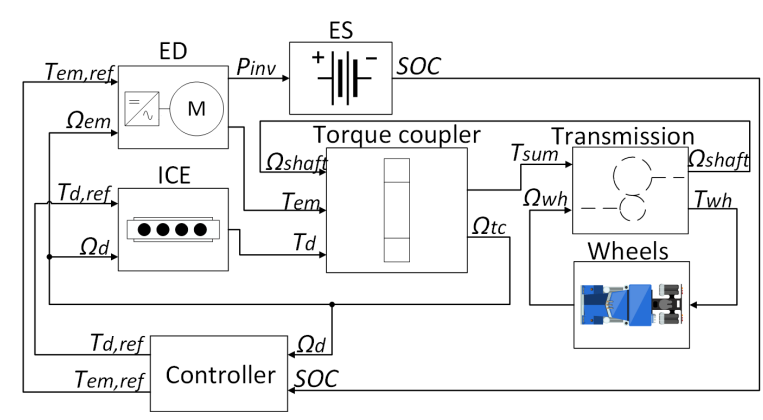

Figure 1: Layout example of parallel hybrid model

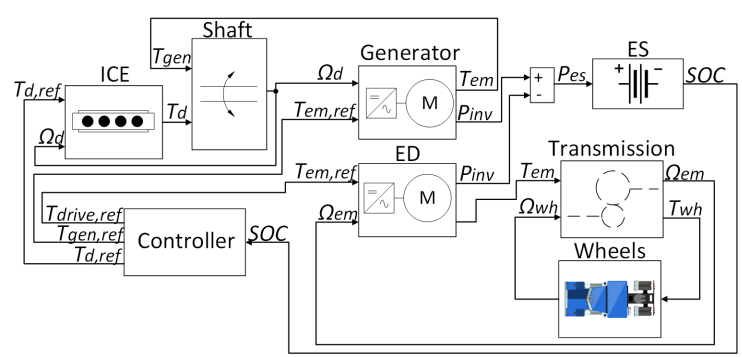

Figure 2: Layout example of series hybrid model

\section{Internal combustion engine model}

A diesel engine is a primary prime mover for off-road machines. The modeling of it is a complex task since an ICE consists of different subsystems and dynamics. Thus, there are several techniques to model a diesel engine. The map-based model was used for simulation due to its relative simplicity and fast computational time (Winke, 2019). Furthermore, ICE representation by an efficiency map is an effective methodology when the question of energy management and system analysis is considered. Lauren et al. (-) has developed a relatively simple calculation schema to obtain brake specific fuel consumption or efficiency maps from curves of the engine manufacturer. However, the map-based model considers only the crankshaft dynamics neglecting the air and fuel path dynamics. Purely map-based model approach results in the immediate reaction of ICE torque to changes, while the torque response is non-instantaneous. Besides, different dynamics determine the resultant engine torque changes under torque increase and decrease. During sudden cutdown in load torque, the fuel reduction dictates the torque decrease. While at torque rise, the turbocharger does not respond fast enough to meet air requirements (Hiereth and Prenninger, 2003), whereas fuel could be injected quickly to satisfy torque demand, leading to the low air to fuel ratio that would cause sooting. Hence, the efficiency map is coupled with estimated dynamics to limit the changes in torque. The dynamics consist of 


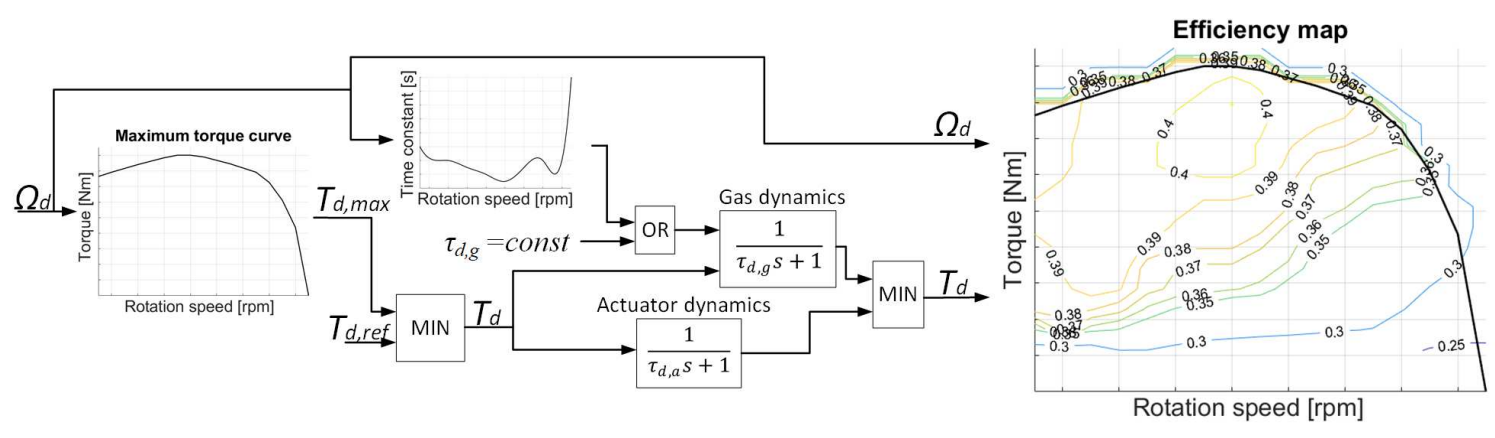

Figure 3: Block diagram of diesel engine model

gas, actuator, and crankshaft dynamics. The actuator dynamics include all delays related to the fuel injection control, fuel rack, and injection delays, and, if variable geometry or waste-gate turbocharger is incorporated, the dynamics of its control. The latter is included in actuator dynamics since the torque reduction arises almost as rapidly as fuel is cut down. The gas circulation dynamics comprise intake manifold, exhaust manifold, and turbocharger dynamics.

In the proposed model presented in Figure 3, gas and actuator dynamics are modeled by the first-order transfer function and are considered in parallel. Thus, the torque reference (fuel rack position) of an ICE is determined by the control strategy. The simulated output torque is limited by the maximum torque curve and delayed by the first-order time constant $\left(\tau_{d, a}\right.$ and $\left.\tau_{d, g}\right)$, that can be represented as a function of the rotational speed. The minimum value representing the torque changes is chosen from the actuator and gas dynamics transfer function blocks. The use of two transfer functions ascertains the realistic behavior of the machine during torque increase and decrease. The response of the diesel engine to a step function is presented in Figure 4 .

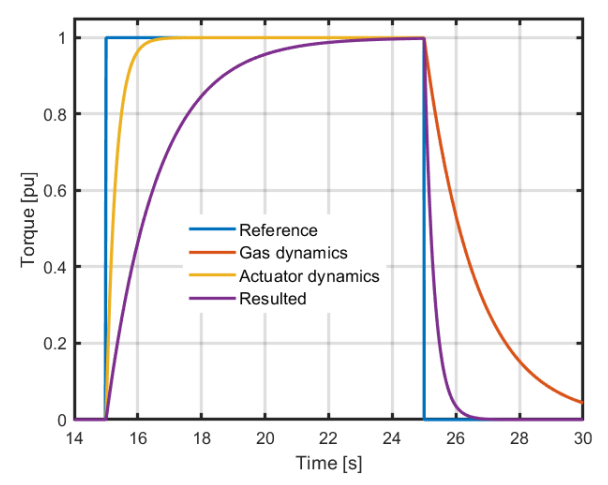

Figure 4: Step response of diesel engine

The representation of diesel engine dynamics with the second-order transfer function and dead time has been shown to provide more accurate results than the first-order model applied in this study (Lindh and Nevaranta, 2020). However, achieving advantages of the second-order system requires empirical results to determine unknown parameter values of the transfer function.

The crankshaft dynamics is considered for the series hybrid model alone, and it is modeled:

$$
\Omega_{d}=\int \frac{T_{d}-T_{l o a d}}{J_{d}} d t
$$

where $J_{d}$ rotational inertia of the diesel engine genset (series hybrid), $\Omega_{d}$ angular velocity of the ICE, $T_{d}$ torque of the ICE, $T_{\text {load }}$ load torque. While in the parallel model, the inertia of the ICE is neglected due to the low inertia of the diesel engine in comparison with the total vehicle mass. The ICE rotational speed is calculated from the velocity of wheels.

The FC of a diesel engine for the given torque and speed is calculated in accordance with the ICE efficiency (Heywood, 1988):

$$
F C=\frac{P_{f u e l}}{E_{J}}=\frac{T_{d} \Omega_{d}}{E_{J} \eta_{d}},
$$

where $P_{f u e l}$ - total power from fuel, $E_{J}$ fuel heat value. Typically, it is in a range 42.7 to $43.1 \mathrm{MJ} / \mathrm{kg}, \eta_{d}$ efficiency of engine obtained from efficiency map.

\section{Electrical drive model}

The model of the electrical drive, presented in Figure 5, comprises EM and inverter models. To appraise the suitability of the components, the model should capture if the electrical drive has sufficient capacity to provide the required power. Power losses should be estimated to evaluate the energy efficiency of the electrical drive. Further, the model should be applicable to test different machine types.

An EM can be modeled quasi-statically or dynamically. The dynamic model uses the space-vector model 


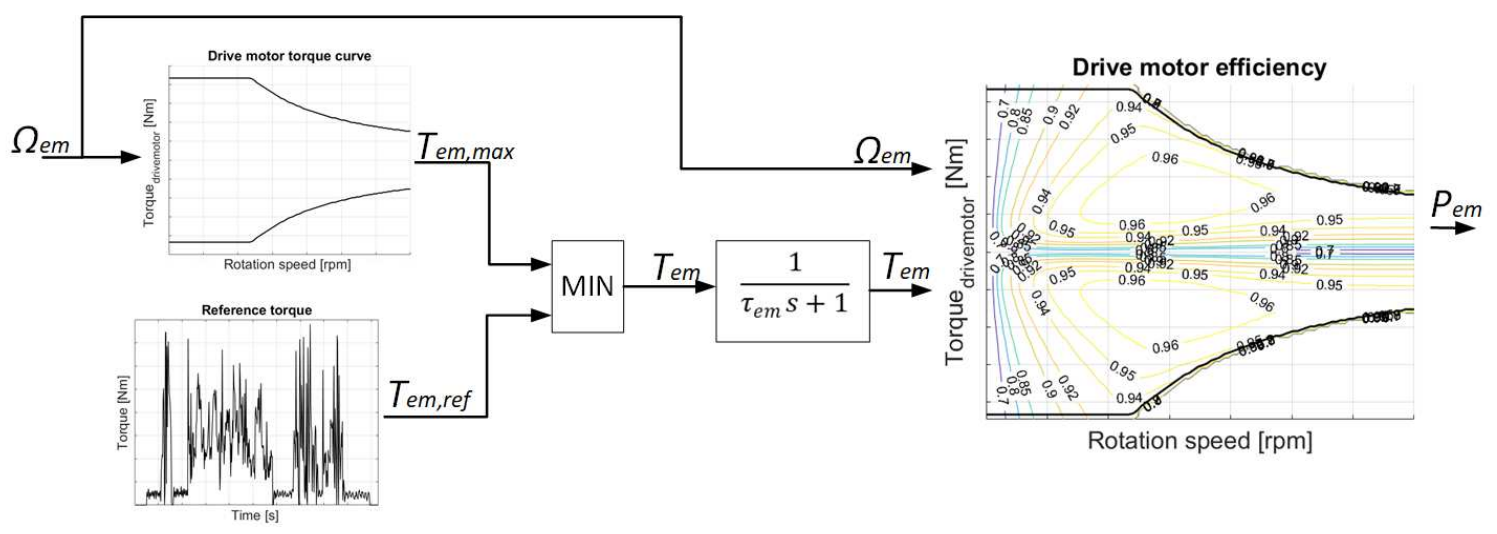

Figure 5: Block diagram of electrical drive model

of the EM in 2-axis coordinated system. There are a couple of drawbacks in this approach. Firstly, 2axis models of different motor types are different. Secondly, values of the motor electro-magnetic parameters are required. In addition, the 2 -axis motor model requires a 2-axis model of an inverter with the vector control, and that control also differs by motor type. Especially the requirement of known motor parameters complicates the modeling task. Parameters can vary in large scale depending on the motor design goals. Thus, this is not a feasible solution for the proposed simulation tool. Instead, the electrical machine is simulated using the quasi-static approach presented in Figure 5, where the efficiency map is used to describe the losses in the machine over the torque-speed range and the maximum torque curve to bound torque changes. Typically, the efficiency map can be provided by an EM manufacturer, or if the EM is self-manufactured the efficiency map may be achieved by conducting few measurements. The power losses of the EM can be determined at standardized load points according to standard IEC 61800-9. The power losses estimation at additional points can be done with the interpolation method. The efficiency map is then calculated from the resulted power losses. The map is coupled with the first-order time constant model.

In the hybrid vehicle application an electric motor is controlled by a frequency inverter. For the initial design purposes, an average efficiency is used to estimate inverter losses since the efficiency of the inverter varies only slightly over the range of the load cycle. The inverter model is included in the EM model and represented by the constant efficiency and the first order transfer function. Of course, both the inverter and the electric motor torque dynamics are in the millisecond time range. However, in real applications, torque rise time is always limited by either the inverter torque controller or by torque ramping. This is done to protect mechanical parts. It is required to present that lag in co-simulations with an MBD simulator (Lindh, 2013). When load cycles are used for dimensioning, one should have small enough time constant in order to follow the reference load cycle.

In the electrical drive mode, the reference torque is determined by the hybrid powertrain supervisory controller, limited by the maximum torque curve, and delayed by the first-order time constant Figure 5. The requested electrical power is calculated considering the direction of the power flow and relevant efficiency values of the components:

$$
\begin{gathered}
P_{E M}=T_{E M} \Omega_{E M} \\
P_{\text {in }, \text { drive }}=\frac{P_{E M}}{\eta_{E M} \eta_{\text {inv }}}, P_{E M} \geq 0 \\
P_{\text {in }, \text { drive }}=P_{E M} \eta_{E M} \eta_{\text {inv }}, P_{E M}<0,
\end{gathered}
$$

where $T_{E M}$ EM torque,$\Omega_{E M}$ EM angular velocity, $\eta_{E M}, \eta_{i n v}$ efficiency of the EM and the inverter. The positive value of power corresponds to the direction from the EM to the transmission.

\section{Energy storage model}

Electrical ES is a secondary energy source in the HEV. Rechargeable batteries or ultracapacitors (UC) are used as ES in a hybrid system. Battery energy storage (BES) is characterized by high energy density but has relatively low power density. A UC is capable of supplying a large instant power, although it has a much lower energy density (Burke, 2007). The battery is connected directly to the DC link of the vehicle. Therefore, the voltage level of the storage should meet the requirements of the DC bus. In such topology, the losses that would be caused by an additional DC converter are avoided. Since the voltage level of a UC varies greatly with varying SOC, the UC is connected via a DC converter in order to supply a constant voltage. 


\section{Battery energy storage model}

The Li-ion battery technology is widely applicable for HEV (Ding et al., 2019). Therefore, this type of battery was utilized for simulation. The model is based on the Simscape Electrical battery model described by Shepherd equations (Tremblay and Dessaint, 2009), which discern a generic dynamic of a rechargeable battery. The voltage on the output terminals $\left(E_{b}\right)$ is calculated by:

$$
\begin{gathered}
V_{b}=E_{0}-R I_{b} \\
E_{b}=V_{b}-\frac{K Q_{m}}{Q_{m}-Q} Q-\frac{K Q_{m}}{Q_{m}-Q} i_{b *}+A e^{B Q} \\
i_{b *} \geq 0 \\
E_{b}=V_{b}-\frac{K Q_{m}}{Q_{m}-Q} Q-\frac{K Q_{m}}{Q+0.1 Q_{m}} i_{b *}+A e^{B Q} \\
i_{b *}<0
\end{gathered}
$$

where $K$ is polarization constant/polarization resistance $[\mathrm{V} / \mathrm{Ah}] /[\Omega], E_{0}$ is constant voltage value, $A$ is exponential zone amplitude $[\mathrm{V}], B$ is exponential zone time constant inverse $\left[\mathrm{Ah}^{-1}\right], Q_{m}$ is maximum battery capacity, $R$ is internal resistance. Parameters are extracted from any particular manufacturer discharge curve. $i_{b *}$ is filtered current, $Q$ is actual charge:

$$
Q=\left(1-S O C_{i n i t}\right) Q_{m}+\int \frac{I_{b}}{3600} d t
$$

The required power of ES $P_{e s}$ is used as an input signal to the block. Then, based on the voltage value on the output terminals, the current of the battery is calculated:

$$
I_{b}=\frac{P_{e s}}{E_{b}} .
$$

The SOC is estimated by modified Coulomb counting method:

$$
S O C=S O C_{i n i t}+\int \frac{I_{b}}{3600 Q_{m}} d t .
$$

\section{Ultracapacitor model}

A simple RC-circuit model was used to model a UC since the data for the model can be obtained from datasheets of a manufacturer. The voltage on the output terminals of the UC is calculated by:

$$
U_{U C}=\frac{1}{C} \int I_{U C} d t+I_{U C} R_{E S R}
$$

where $C$ is capacitance of the $\mathrm{UC}, R_{E S R}$ is equivalent series resistance, $I_{U C}$ is the current of the UC.

Similarly to the BES model, the model of the UC should also describe its SOC, which is defined by terminal voltage and the maximum voltage
$\left(U_{U C, \max }\right)$ (Ceraolo et al., 2017):

$$
S O C=\frac{U_{U C}^{2}}{U_{U C, \max }^{2}},
$$

Losses in the DC/DC converter are evaluated by an average efficiency.

\section{Torque coupler model}

A torque coupler is an essential component in the parallel hybrid system. It is aimed to couple and transmit mechanical power from a diesel engine and an EM to the wheels, or to divide the power from an ICE between traction wheels and ES (Ehsani et al., 2004). The output torque is a linear superposition of two separate torques, where $k_{1}$ and $k_{2}$ are the constants defined by the geometry of the torque coupling device and $\eta$ is the efficiency of the coupler.

$$
\begin{aligned}
& T_{\text {sum }}=T_{d} k_{1} \eta_{1}+T_{E M} k_{2} \eta_{2}, T_{E M} \geq 0 \\
& T_{\text {sum }}=T_{d} k_{1} \eta_{1}+\frac{T_{E M} k_{2}}{\eta_{2}}, T_{E M}<0
\end{aligned}
$$

The required output torque to drive wheels can be adjusted by applying different torques from an ICE and an electrical drive. While the angular velocity cannot be controlled freely, both machines will rotate with the speed described by:

$$
\begin{gathered}
\Omega_{\text {shaft }}=\frac{\Omega_{d}}{k_{1}}=\frac{\Omega_{E M}}{k_{2}} \\
\text { if } k_{1}=k_{2}=1: \Omega_{\text {shaft }}=\Omega_{d}=\Omega_{E M}=\Omega_{t c}
\end{gathered}
$$

\section{Transmission model}

The transmission consists of the gearbox and the mechanical driveline and it connects the wheels to the propulsion system. The mechanical torque transferred from the driving block (a torque coupler for parallel topology and an EM for series hybrid Figure 1 and Figure 2) to the wheels considering transmission $\left(i_{\text {diff }}\right)$ and gearbox $\left(i_{\text {gear }}\right)$ ratios, and their efficiencies $\left(\eta_{\text {gear }}, \eta_{\text {diff }}\right)$, can be represented by an equation dependent on the direction of the torque:

$$
\begin{gathered}
T_{w h}=T_{\text {in }} i_{\text {gear }} i_{\text {diff }} \eta_{\text {gear }} \eta_{\text {diff }}, T_{i n} \geq 0 \\
T_{w h}=\frac{T_{\text {in }} i_{\text {gear }} i_{\text {diff }}}{\eta_{\text {gear }} \eta_{\text {diff }}}, T_{i n}<0
\end{gathered}
$$

The angular velocity of the rotational shaft is:

$$
\Omega_{\text {gear_in }}=\Omega_{w h} i_{g e a r} i_{d i f f}
$$

where $\Omega_{w h}$ is rotational velocity of the wheels. 


\section{Wheels model}

The force and angular velocity of wheels are calculated according to:

$$
F_{w h e e l}=\frac{T_{w h}}{r_{w h e e l}}, \Omega_{w h}=\frac{v_{v e h}}{r_{w h e e l}},
$$

where $r_{\text {wheel }}$ radius of wheels.

\section{Load cycle}

A load cycle describes the power of the mobile machine during the operational process. The dimensioning is based on the load cycle data, and they are used as input to the simulation tool. The origin of the duty cycle can be a recorded load cycle given by a manufacturer, standard loading curves from (EPA, 2021) - Figures 10 and 15, or external simulators. The reference torque and speed curves emulating mobile working machine actions can be obtained by multibody dynamics (MBD) simulation.

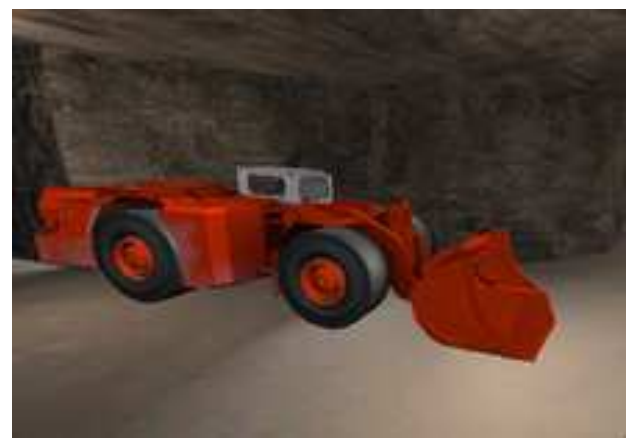

Figure 6: A screenshot from the MBD simulator depicting a virtual mine loader in a virtual mine.

MBD simulation is a numerical simulation method which models the simulated system as a series of interconnected flexible or rigid bodies. These bodies are interconnected by mechanical constraints, forces, etc. Real-time version of an MBD simulation allows to drive a realistic machine in a realistic environment, making it a good tool for virtual prototyping of mobile machinery. There are two options to use load cycles from an MBD: co-simulation, where the power train is modeled in Simulink and the rest in an MBD simulation, or record load cycle data from an MBD simulator. The communication between the MBD simulator and Simulink is implemented via a TCP/IP connection (Montonen et al., 2012) (Lindh et al., 2014) (Montonen and Lindh, 2014). An interval of $2 \mathrm{~ms}$ is used for the communication between Simulink and the MBD simulator, since the MBD model used has limitations for the maximum step time. Exceeding this limitation renders the model unstable. This co-simulation scheme facilitates the comparison of different topologies and component sizes for the power train and verifying results obtained by the dimensioning tool. A mine loader in a virtual mine was considered for the co-simulation Figure 6 (Nokka et al., 2017).

\section{Simulation principle of the model}

The simulation principle of the introduced model depends on the type of loading data used. If it is recorded load cycle or curves obtained from EPA, then the principle is represented in Figure 7 and Figure 8a. The loading torque from driving blocks is transmitted to the wheels, while the measured or simulated speed of the vehicle is used as input to the wheels model and transmitted from the wheels to the driving blocks. The power consumption of the system can be determined at every simulation step as well as the energy balance of the system.

The calculated power request of the mobile machine must include the power consumed by the hydraulics drive. If it is known, hydraulics can be represented as a power or torque-speed curves. Therefore, the power produced by the ICE and the ES is divided between drivetrain and hydraulics. For the parallel hybrid Figure 1 , the hydraulics can be connected to the torque coupler, where the power of hydraulics $P_{h y d}$ and power balance is determined as:

$$
\begin{gathered}
T_{\text {sum }} \Omega_{\text {shaft }}+P_{\text {hyd }}=P_{d} \eta_{1}+P_{E M} \eta_{2}, P_{E M} \geq 0 \\
T_{\text {sum }} \Omega_{\text {shaft }}+P_{\text {hyd }}=P_{d} \eta_{1}+\frac{P_{E M}}{\eta_{2}}, P_{E M}<0
\end{gathered}
$$

For series topology, hydraulic actuator can be powered by separate electrical drive as it will be shown later.

For the Simulink model coupled with the MBD simulator, the simulation principle is provided in Figure $8 \mathrm{~b}$. The simulator is driven by a human driver. The required reference torque is exported from the MBD simulator $\left(T_{r e f}\right)$ to the proposed simulation tool. The model of electrical drive calculates the simulated torque back to the MBD simulator, which returns the speed of the vehicle. The controller in the Simulink manages the power generation and power balance in the system. In the MBD, the tractive and hydraulic drivelines are decoupled and powered by two separate electrical drives. The torque required by the hydraulics is used as reference torque for the EM, while the simulated torque and the angular speed are inputs to the MBD. For the traction drive, the outputs of the MBD are the required loading torque and angular rotational velocity of the machine. 


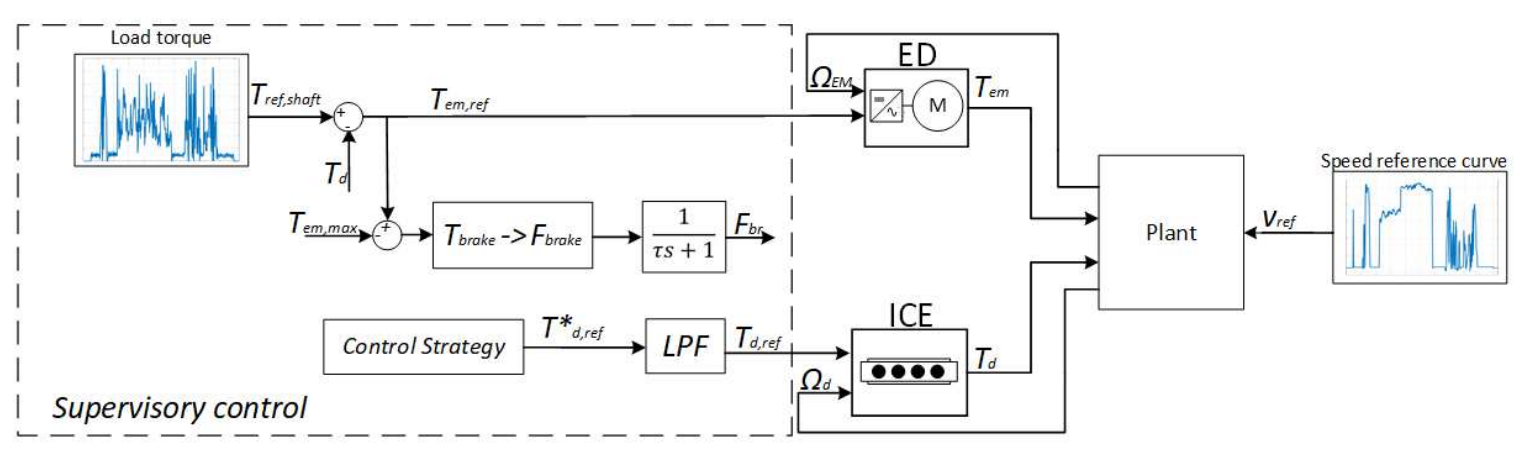

Figure 7: Simulation principle and supervisory control of the parallel HEV

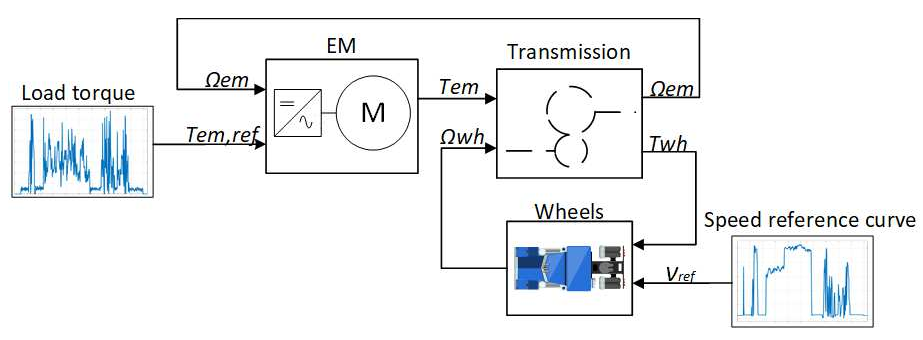

(a)

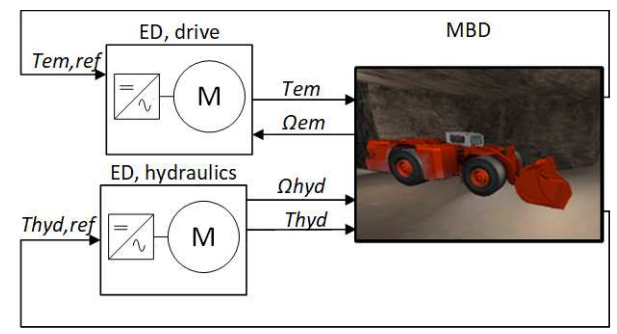

(b)

Figure 8: Simulation principle of series HEV for a) recorded loading cycles and b) for MBD simulator

\section{Control principle of HEV}

The supervisory controller allocates the required loading power between the ICE and the ES, where the diesel engine loading is dictated by a control strategy (CS). The rule-based CS (Power Flow Control Strategy (PFCS) and Thermostat Control Strategy (TCS) (Shabbir and Evangelou, 2016), and Electric Assist Control Strategy (EACS) (Johnson et al., 2000)) were used for the simulation due to their simplicity since the focus of this work is not to optimize the efficiency and performance of components but to estimate the initial dimensions of the elements.

\section{Parallel hybrid}

The supervisory control principle of the parallel hybrid system includes the following key functions: loading and regenerative braking control Figure 7. CS defines the ICE reference torque value, and then it is low pass filtered (Ottosson, 2007) to reduce the reaction of the engine to load changes. The requisite electrical drive reference torque is determined by subtracting the torque value of the diesel engine from the total reference torque. If the electrical drive braking capacity is not sufficient, mechanical braking is used. The torque of the mechanical braking system is determined by subtracting the EM maximum braking torque value from the required total braking torque. The time response of the mechanical braking system is modeled by trans- fer function with a time lag coefficient $(\tau=0.01)$ et al., 2016).

\section{Series hybrid}

The traction drive in the series hybrid is decoupled from the diesel engine. The full loading torque is provided by electrical drive or distributed between several electrical drives depending on the application. Thus, the required torque is the input to the EM Figure 8. The genset control block determines the reference torque values for the ICE and the generator Figure 9. The calculated diesel engine power reference from CS is first low pass filtered. Then, the reference value of the engine torque for the desired power level is selected according to the maximum efficiency point. Based on power and torque values, the diesel engine genset speed is determined. The reference torque to reach the target velocity is calculated in the PI controller based on the error between the required and measured angular velocity of the shaft. The reference torque of a generator is then calculated as a difference between the required torque and actual torque produced by the diesel engine.

The duty cycle of off-road machine often has large and quick power variations. Mode of operation can be frequently changing from idling or low load to high power. During idle or low loading, the efficiency of the ICE is poor. Thus, those operating points should 


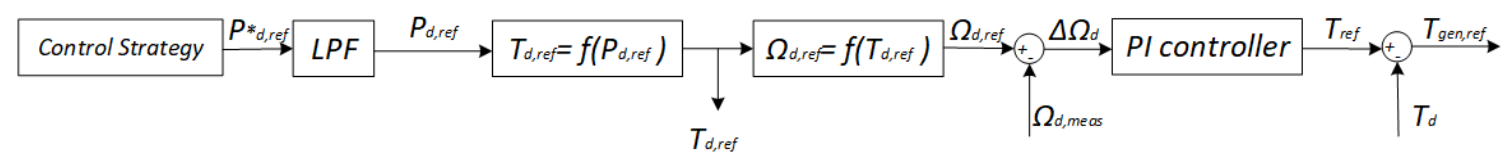

Figure 9: Supervisory control of series hybrid

be avoided. However after full loading, when the temperature of the engine reaches high values, the engine needs to cool down for a predetermined amount of time typically idling 3 to 5 minutes before being shut down (Keel-Blackmon et al., 2016). Typically the engine lubrication will cease when the engine is stopped, which may lead to overheating of the oil and cause additional wear to the internal components of the engine (Rundo, 2015) (Polichronis et al., 2013). Thus, idling is necessary for even cooling, reducing wear, and preventing coking of oil. However, if the oil flow is powered by an auxiliary electric pump, the engine can be stopped shortly. In the framework of this work, during low load, a diesel engine is stopped after a predetermined amount of time.

\section{Simulation results}

This section describes case studies of dimensioning an HEV to demonstrate the applications of the simulation tool. Backhoe loader and excavator are simulated using a standardized curve with series and parallel hybrid topologies. Then an underground mine loader is simulated using a load cycle obtained by an MBD simulation. Only series hybrid version of the mine loader is simulated.

\subsection{Case study 1: Standardized curve of backhoe loader}

The dimensioning of a hybrid power train was implemented to standardized loading curves of the backhoe loader (EPA, 2021). The provided speed and torque values are acquired from a diesel engine crankshaft Figure 10. Therefore, the transmission is not modeled. It is assumed that all the power is allocated to the driveline since the power consumed by hydraulics cannot be distinguished from the driveline power. The load cycle is characterized by considerable torque variations. For the parallel hybrid layout, the full load is connected to the shaft powered by ICE and electrical drive. For a series hybrid, the total load power is provided by a single electrical drive.

The selection of components for different options is presented in Table 1. Scania and AGCO off-road diesel engines were used for simulation (Scania, -) (AGCO,
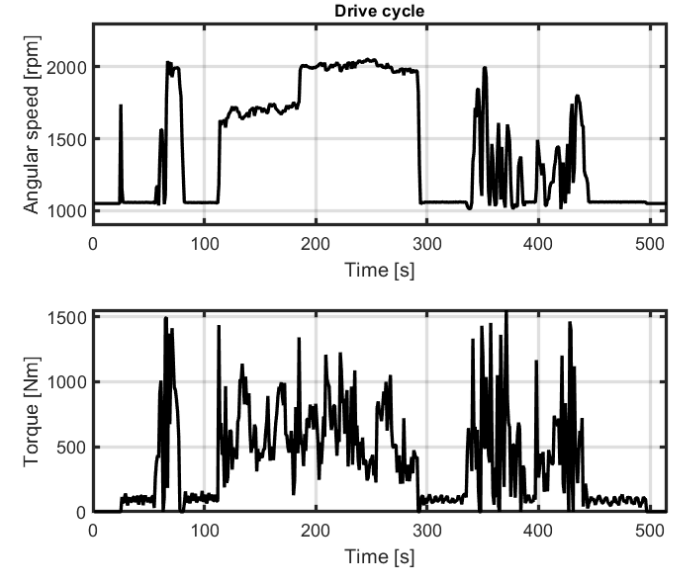

Figure 10: The load cycle of the backhoe loader

2008). The data for AGCO $148 \mathrm{~kW}$ engine were obtained from Turku University of Applied Science, Engine and Powertrain laboratory and are not publicly available. Induction motors (IM) and permanent magnet $(\mathrm{PM})$ generators were chosen for the EMs. The EM efficiency map is engineered by calculating the motor losses, which are comprised from winding (copper), iron, mechanical and additional losses. Fraction of these losses for IE3 classified inverter fed induction motors are reproduced according to (Aarniovuori et al., 2018). Major part of losses consists of winding losses. These vary in large scale with the speed and torque. Winding losses are interpolated from the data of the manufacturer $(A B B, 2021)$ at speed range from zero to rated speed and in the field weakening area the dependency of speed and torque is comprised as explained in (Doppelbauer, 2020). Part of the iron losses is present in the whole speed range (as in a no-load situation) and part is frequency dependent. The iron losses are therefore calculated in two parts. As speed increases the iron losses increase until the rated speed is achieved and above that, in field weakening, the share of iron loss is decreasing (Doppelbauer, 2020). The last loss components in this EM efficiency map are the additional and mechanical losses, which increase as the speed increases. A UC was utilized for the parallel hybrid topology (Eaton, 2019), and a BES for the series hybrid (Altairnano, 2021). To meet the requirements 
for the flow of current, the BES was over-dimensioned. Control strategies maintain the SOC of ES at the designated level. Therefore, the SOC at the beginning of a driving cycle and at the end are equal. The load cycle is followed for all dimension variants presented.

Table 1: The component dimensions of the backhoe loader

\begin{tabular}{ccccc}
\hline & \multicolumn{2}{c}{ Parallel } & \multicolumn{2}{c}{ Series } \\
\hline Manufacturer & Scania & AGCO AGCO & AGCO \\
ICE rated power $[\mathrm{kW}]$ & 202 & 148 & 148 & 129 \\
ICE idle power $[\mathrm{kW}]$ & 20 & 15 & 15 & 13 \\
Electric motor $[\mathrm{kW}]$ & 132 & 132 & 250 & 250 \\
type & $\mathrm{IM}$ & $\mathrm{IM}$ & $\mathrm{IM}$ & $\mathrm{IM}$ \\
Generator $[\mathrm{kW}]$ & - & - & 160 & 132 \\
type & - & - & $\mathrm{PM}$ & $\mathrm{PM}$ \\
ES capacity $[\mathrm{kWh}]$ & 1.3 & 1.3 & 36.25 & 36.25 \\
type & $\mathrm{UC}$ & $\mathrm{UC}$ & $\mathrm{BES}$ & $\mathrm{BES}$ \\
\hline
\end{tabular}

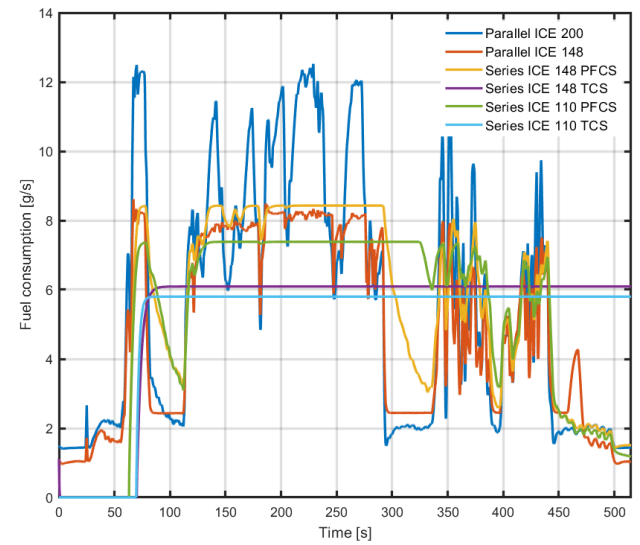

Figure 11: Fuel consumption of various hybridization options of the backhoe loader

The fuel consumption for various hybridization options is presented in Figure 11. It can be noticed that for series hybrid, the FC changes bear a more stable pattern, while the parallel layout exhibits more substantial fluctuations. The fuel and energy consumption obtained from the simulation results are provided in Table 2. The overall efficiency is calculated as follows, where $E_{\text {fuel }}$ is the produced energy and $E_{\text {loss }}$ is the loss of energy:

$$
\eta=\frac{E_{\text {fuel }}-E_{\text {loss }}}{E_{\text {fuel }}} 100 \% .
$$

The initial estimation suggests that the parallel hybrid topology with the $148 \mathrm{~kW}$ diesel engine provides the most efficient solution. In this case, the ICE is working close to the optimal points about $35 \%$ of the time. However, the proposed methodology does not consider the increase in FC during transient operation modes of the ICE, which has a more significant effect on the parallel configuration than on the series configuration. The rate of change in the simulated torque varies from -9 $5.63 \% / \mathrm{s}$. This can be taken into account with a correction factor (Lindgren, 2005) calculated from the synthetic transient data measurements, which is not achievable with the available data.

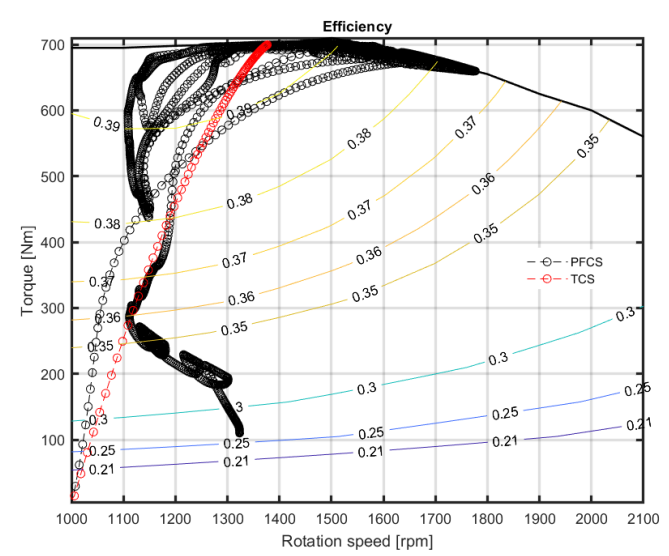

Figure 12: Working points of ICE with TCS (red dots) and PFCS (black dots) on diesel-generator efficiency map

Table 2: Simulation results of the backhoe loader with different topologies and control strategies (CS): EACS - Electric Assist CS, TCS - Thermostat CS, PFCS - Power Flow CS

\begin{tabular}{ccccc}
\hline & \multicolumn{2}{c}{ Parallel } & \multicolumn{2}{c}{ Series } \\
\hline & \multicolumn{2}{c}{ Scania AGCO } & AGCO & AGCO \\
CS & EACS & EACS & PFCS/TCS & PFCS/TCS \\
\hline FC $[1 / h]$ & 22.84 & 19.97 & $21.86 / 21.84$ & $21.6 / 20.9$ \\
$E_{\text {loss }}[k W h]$ & 21.32 & 17.84 & $20.84 / 20.83$ & $20.79 / 19.78$ \\
$E_{\text {fuel }}[k W h] 32.82$ & 28.74 & $31.48 / 31.47$ & $31.11 / 30.08$ \\
$\eta[\%]$ & 35 & 37.9 & $33.8 / 33.8$ & $34.2 / 34.25$ \\
\hline
\end{tabular}

The second best solution is series hybrid with the $129 \mathrm{~kW}$ engine. The series hybrid option is less efficient since additional losses are introduced by additional components and full power transfer through the electric motor.The FC of series hybrid depends on the CS chosen - Figure 12. TCS (red dots) provides more fuel-efficient results than PFCS since the ICE always operates in the most efficient point. Using PFCS (black dots), power of the ICE varies to maintain SOC of the 
battery close to the designated level - Figure 13. In addition, the increase in FC during the start/stop of the ICE is not considered. However, CSs produce a low engine start count and therefore it can be neglected. Furthermore, the engine is needed to idle after working at the maximum torque. Due to idling, the additional FC results in approximately 0.081-0.24 l (70-200 g) . The series hybrid with the $148 \mathrm{~kW}$ ICE is oversized. The engine is not able to work in the most efficient points regardless of the different CSs used. The Figure 14 depicts that the $202 \mathrm{~kW}$ engine also is oversized for a parallel hybrid setup.

The best fitting size of diesel engine can be determined visually by plotting an efficiency map together with the working points, see Figures 12 and 14. Such graphical representation allows to illustratively inspect and scale the suitable power level of the components. The calculated parameters can be depicted to assist in the selection process. For instance, the SOC of the battery for series hybrid with the $129 \mathrm{~kW}$ ICE during load cycle is provided in Figure 13. Based on the SOC variations, the lifetime of the battery can be evaluated (Immonen, 2013). Therefore, the calculation of parameters of interest (such as fuel consumption and power losses) together with the visual representation facilitate the scaling of the mobile $\mathrm{HEV}$ and provide a useful tool for the dimensioning.

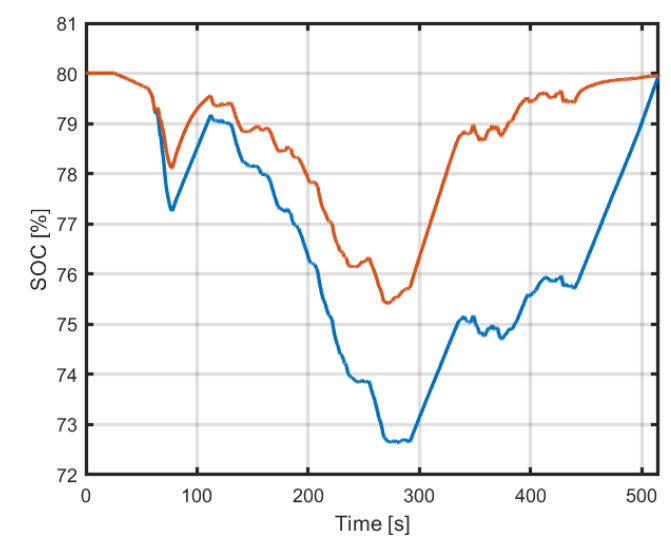

Figure 13: SOC of the battery with TCS - blue curve and PFCS - red curve

\subsection{Case study 2: Standardized curve of excavator}

To represent different hybridization examples, the standardized load cycle of an excavator (Figure 15) was considered. This cycle does not include high peak power modes. Most of the time the cycle exhibits steady behavior with a number of operating modes

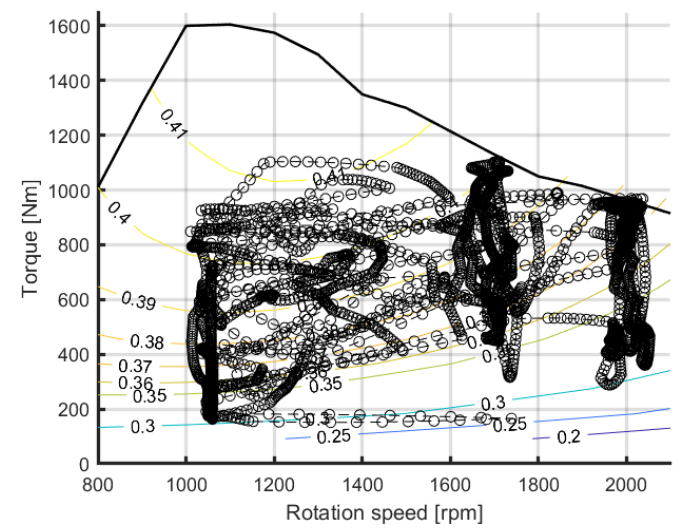

Figure 14: Working points of parallel hybrid with 202 $\mathrm{kW}$ engine

with low torque requirement. The average power consumption is close to the peak power, and the power range of the diesel engines does not differ significantly. When the diesel engine power is close to the maximum power, the $\mathrm{UC}$ balances the torque variation. If the maximum power produced by the ICE is less than the maximum load, a larger capacity of ES is needed to provide the required additional power. As for the previous case, the SOC of $\mathrm{ES}$ is equal at the start and end of the cycle and the predetermined driving cycle is followed. Three choices for the hybrid powertrain unit are presented in Table 3. The data for the $180 \mathrm{~kW} \mathrm{ICE}$ is obtained from DLG (2013).
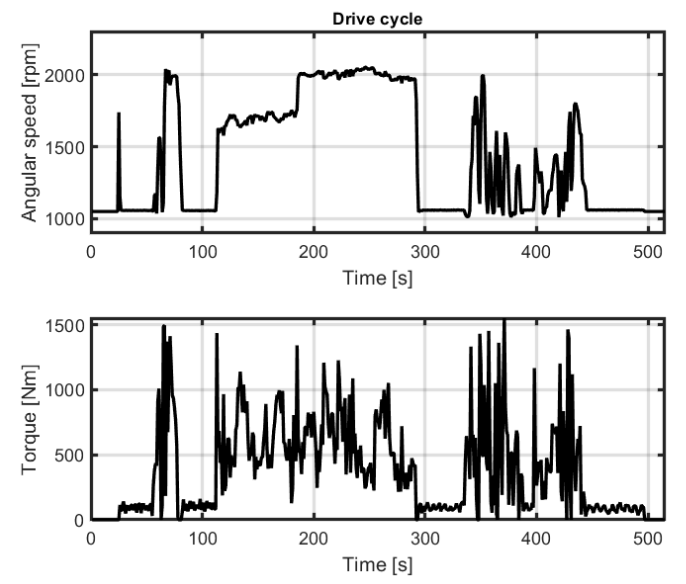

Figure 15: The load cycle of the excavator

The results of simulated variants (Table 4) show that the FC is minimized for parallel configuration with the $180 \mathrm{~kW}$ diesel engine. However, this option requires ES with a bigger capacity. And furthermore, the difference in FC compared to the option with the $202 \mathrm{~kW}$ ICE 
Table 3: The component dimensions of the excavator

\begin{tabular}{cccc}
\hline & \multicolumn{2}{c}{ Parallel } & Series \\
\hline Manufacturer & Scania & FPT & FPT \\
ICE rated power $[\mathrm{kW}]$ & 202 & 180 & 180 \\
ICE idle power $[\mathrm{kW}]$ & 20 & 18 & 18 \\
Electric motor $[\mathrm{kW}]$ & 75 & 75 & 200 \\
type & $\mathrm{IM}$ & $\mathrm{IM}$ & $\mathrm{IM}$ \\
Generator $[\mathrm{kW}]$ & - & - & 200 \\
type & - & - & $\mathrm{PM}$ \\
ES capacity $[\mathrm{kWh}]$ & 1.3 & $6.5 / 36.25$ & 36.25 \\
type & $\mathrm{UC}$ & $\mathrm{UC} / \mathrm{BES}$ & $\mathrm{BES}$ \\
\hline
\end{tabular}

is not substantial. Even though the increase in FC is not considered during transient operation modes, those operating regions are neither frequent nor broad for the load cycle under consideration. The power losses in a series hybrid topology negate the benefits of working in the most efficient points of the diesel generator set (Figure 16), which is reflected by the resulted FC.

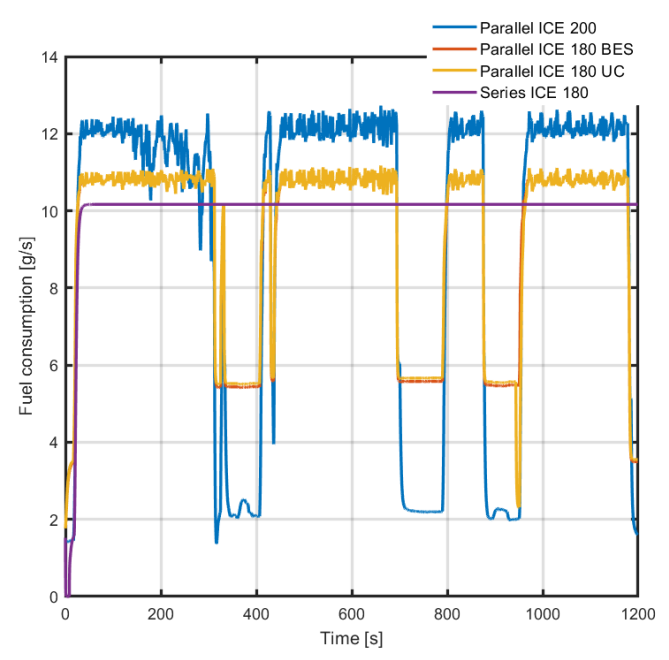

Figure 16: Fuel consumption of various hybridization options of the excavator

\subsection{Case study 3: Underground loader}

Different hybridization options were considered for the underground mine loader from the MBD simulation tool (Nokka et al., 2017). The diesel-powered machine model was verified by measurements done with the actual machine (Nokka, 2018). The model includes the modeling of transmission and hydraulics, where the input signal to the transmission is the supplied torque, and the output signal is the velocity of the traction motor of the working machine. The input to the hydraulic
Table 4: Simulation results of the excavator

\begin{tabular}{cllc}
\hline & \multicolumn{2}{c}{ Parallel } & Series TCS \\
\hline & Scania & \multicolumn{1}{c}{ FPT } & FPT \\
\hline FC $[\mathrm{l} / \mathrm{h}]$ & 39.23 & $38.99 / 39.1$ & 41.8 \\
$E_{\text {loss }}[k W h]$ & 83.66 & $83.48 / 83.76$ & 93.16 \\
$E_{\text {fuel }}[k W h]$ & 131.38 & $130.55 / 131.03$ & 140.1 \\
$\eta[\%]$ & 36.32 & $36.05 / 36.7$ & 33.5 \\
\hline
\end{tabular}

block is rotational speed and output is the required torque. When hydraulics are powered by the EM, the constant rotational speed is maintained, and the output torque from hydraulics is the reference torque to an EM. The output torque from the MBD simulator includes the torque associated with mechanical losses to maintain constant rotational speed. The load cycle of the underground loader was obtained by a human driver operating the machine (Figure 17). It consists of a starting, driving to the pickup point, filling the bucket, returning to the dump point, and dumping action sets.

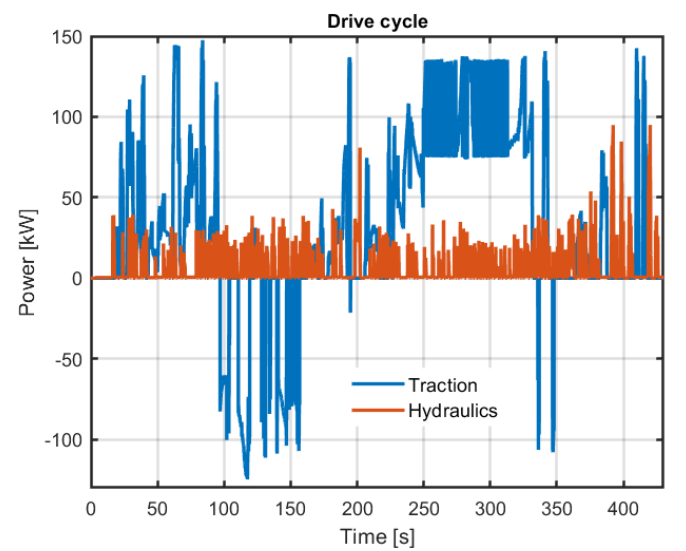

Figure 17: The load cycle of the underground loader

The MBD simulator is coupled with the drivetrain model in the Simulink environment, the components of which are presented in Table 5. For the load cycle under consideration, only series hybrid topology was considered due to several features of the cycle. Firstly, the EM should be able to recuperate energy as much as possible during the downhill movement. That leads to requirement of a bigger ES capacity to store all the energy, as well as a larger EM. Secondly, the cycle is featured by frequent stops during the bucket loading and unloading of the working machine. Therefore, during stop time, the diesel engine will be decoupled from the main shaft to maintain working operational speed. Meanwhile, the hydraulics pump is connected directly to the diesel engine shaft. Thus, the specifica- 
Tupitsina et al., "Simulation tool for dimensioning power train of hybrid working machine"

Table 5: The component dimensions of the underground loader

\begin{tabular}{ccc}
\hline & \multicolumn{2}{c}{ Series } \\
\hline Manufacturer & AGCO & Perkins \\
ICE rated power $[\mathrm{kW}]$ & 74 & 63 \\
ICE idle power $[\mathrm{kW}]$ & 10 & 10 \\
Drive motor $[\mathrm{kW}]$ & 160 & 160 \\
type & $\mathrm{IM}$ & $\mathrm{IM}$ \\
Hydraulics motor $[\mathrm{kW}]$ & 75 & 75 \\
type & $\mathrm{IM}$ & $\mathrm{IM}$ \\
Generator $[\mathrm{kW}]$ & 75 & 75 \\
type & $\mathrm{PM}$ & $\mathrm{PM}$ \\
ES capacity $[\mathrm{kWh}]$ & 36.25 & 36.25 \\
type & $\mathrm{BES}$ & $\mathrm{BES}$ \\
\hline
\end{tabular}

tions of the ICE should meet the power requirements of the pump. Two of these factors exclude the benefits of the parallel hybrid since the components will be over-dimensioned to satisfy the mobile machine performance. These characteristics are in the favor of series hybrid topology, where the traction and hydraulics are powered by two independent EMs and ICE is mechanically decoupled from the driveline.

The results of the dimensioning are presented in Table 6. The option with the $74 \mathrm{~kW}$ ICE (DLG, 2013) has better fuel economy since the operating point of the diesel genset has higher efficiency than the variant with the $64 \mathrm{~kW}$ engine (DLG, 2013) - Figure 18.

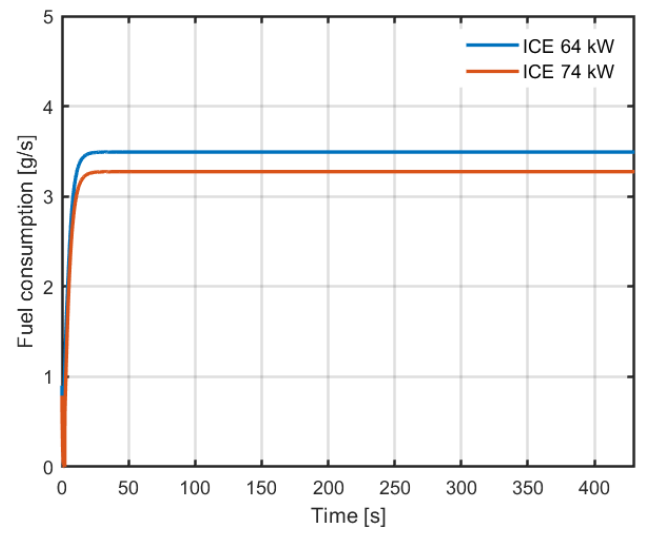

Figure 18: The fuel consumption of the underground loader

\section{Conclusion}

A new simulation tool targeted at the analysis of offroad machinery hybridization was presented. Example
Table 6: The simulation results of the underground loader

\begin{tabular}{ccc}
\hline & \multicolumn{2}{c}{ Series TCS } \\
\hline & AGCO & Perkins \\
\hline FC $[\mathrm{l} / \mathrm{h}]$ & 13.42 & 14.34 \\
$E_{\text {loss }}[k W h]$ & 11.57 & 12.76 \\
$E_{\text {fuel }}[k W h]$ & 17.11 & 18.28 \\
$\eta[\%]$ & 32.38 & 30.2 \\
\hline
\end{tabular}

cases of series and parallel topologies with different dimension choices were simulated. Simulations show the feasibility of the power balance-based simulation tool when selecting a hybrid topology and for the initial dimensioning of the hybrid power train components.

A combustion engine and an electrical drive are modeled by efficiency maps and first-order transfer functions representing their dynamics. The behavior of the ultracapacitor is described by an RC-circuit, and the battery energy system is estimated by mathematical formulas. Simulation blocks use efficiency data from the manufacturers. The idea is to use component models that do not require large measurement-based tuning in the initial design phase. The supervisory control principle manages the torque allocation between the energy sources. The simulations use load cycle data that may either be publicly available cycles or self-recorded data. Alternatively, the model can be cosimulated with the multi-body simulator.

The simulation tool is flexible for the initial design when the capacity and efficiency of different components need to be tested. The effect of changes in the size of components can be analyzed visually and quantitatively. The usage of load cycles for dimensioning allows testing different hybrid topology options for nonhybridized mobile vehicles in a simple way. Considering the diesel engine as the base choice, the dimensions of other components can be determined according to the specifications of the engine. The scaling of the ES is fairly straightforward as it involves only changing the number of cells connected in series and parallel. Efficiency maps of EMs with the same type do not vary considerably. Therefore, in the first iteration, the same map can be used with only scaling the capacity of the electrical machine under consideration. The investigated simulation cases provide vivid examples of the use of the simulation tool and the visibility of the resulted data for comparing hybridization options and helps on component dimensioning. Further development of the tool will possibly lead to optimizationbased dimensioning. 


\section{References}

Aarniovuori, L., Niemel, M., Pyrhnen, J., Cao, W., and Agamloh, E. B. Loss components and performance of modern induction motors. In 2018 XIII International Conference on Electrical Machines (ICEM). pages 1253-1259, 2018. doi:10.1109/ICELMACH.2018.8507189.

ABB. ABB Library. Accessed September. 24, 2021 [Online], 2021. URL https://library.abb.com/.

AGCO. AGCO SISU Power, 3rd generation series, 4cylinder diesel engine, 2008.

Altairnano. 70 amp hour cell. Accessed July. 17, 2021 [Online], 2021. URL https://altairnano.com/.

Burke, A. F. Batteries and ultracapacitors for electric, hybrid, and fuel cell vehicles. Proceedings of the IEEE, 2007. 95(4):806-820. doi:10.1109/JPROC.2007.892490.

Ceraolo, M., Lutzemberger, G., and Poli, D. Stateof-charge evaluation of supercapacitors. Journal of Energy Storage, $2017 . \quad 11: 211-218$. doi:10.1016/j.est.2017.03.001.

Ding, Y., Cano, Z. P., Yu, A., Lu, J., and Chen, Z. Automotive li-ion batteries: Current status and future perspectives. Electrochemical Energy Reviews, 2019. 2:1-28. doi:10.1007/s41918-018-0022-z.

DLG. DLG-Powermix. Accessed September. 15, 2021 [Online], 2013. URL https://www.scania.com/ group/en/home.html.

Doppelbauer, M. Overview interpolation/extrapolation in fieldweakening range and overload range for electrical machines, lecture notes. Karlsruhe Institute of Technology (KIT), Germany, 2020 .

Eaton. Xlr-48 supercapacitor. Accessed July. 17, 2021 [Online], 2019. URL https://www.eaton.com/us/ en-us.html.

Ehsani, M., Gao, Y., Gay, S., and Emadi, A. Modern Electric, Hybrid Electric, and Fuel Cell Vehicles: Fundamentals, Theory, and Design. CRC Press LLC, 2004. doi:10.1201/9781420037739.

Enang, W. and Bannister, C. Modelling and control of hybrid electric vehicles (a comprehensive review). Renewable and Sustainable Energy Reviews, 2017. 74:1210-1239. doi:10.1016/j.rser.2017.01.075.

EPA. EPA nonregulatory nonroad duty cycles. Environmental Protection Agency,Accessed Sep. 17, 2021 [Online], 2021. URL https://www.epa.gov/.
Evangelou, S. and Shabbir, W. Dynamic modeling platform for series hybrid electric vehicles. IFAC-PapersOnLine, 2016. 49:533-540. doi:10.1016/j.ifacol.2016.08.078.

Gao, D. W., Mi, C., and Emadi, A. Modeling and simulation of electric and hybrid vehicles. Proceedings of the IEEE, 2007. 95(4):729-745. doi:10.1109/JPROC.2006.890127.

He, X. and Jiang, Y. Review of hybrid electric systems for construction machinery. $A u$ tomation in Construction, 2018. 92:286-296. doi:10.1016/j.autcon.2018.04.005.

Heywood, J. B. Internal Combustion Engine Fundamentals. McGraw-Hill, Inc, 1988.

Hiereth, H. and Prenninger, P. Charging the Internal Combustion Engine. Springer, 2003.

Huang, Y., Wang, H., Khajepour, A., Li, B., Ji, J., Zhao, K., and $\mathrm{Hu}, \mathrm{C}$. A review of power management strategies and component sizing methods for hybrid vehicles. Renewable and Sustainable Energy Reviews, 2018. 96:132-144. doi:10.1016/j.rser.2018.07.020.

ICCT. European stage V non-road emission standars. The International Council on clean transportation, 2016. URL https://theicct.org/sites/ default/files/publications/EU-Stage-V_ policym\%20update_ICCT_nov2016.pdf.

Immonen, P. Energy Efficiency of a Diesel-Electric Mobile Working Machine. Ph.D. thesis, Lappeenranta University of Technology, 2013.

Ishida, K. and Higurashi, M. Hybrid wheel loaders incorporating power electronics. Hitachi Review, 2015. 64(7):41-45.

Johnson, V. H., Wipke, K., and Rausen, D. HEV control strategy for real-time optimization of fuel economy and emissions. SAE transactions, 2000. 109:1677-1690. doi:10.4271/2000-01-1543.

Keel-Blackmon, K., Curran, S., and Lapsa, M. Summary of OEM idling recommendations from vehicle owners manuals. Technical report, OAK RIDGE NATIONAL LABORATORY, 2016.

Lajunen, A., Sainio, P., Laurila, L., PippuriMkelinen, J., and Tammi, K. Overview of powertrain electrification and future scenarios for nonroad mobile machinery. Energies, 2018. 11(5). doi:10.3390/en11051184. 
Lajunen, A., Suomela, J., Pippuri, J., Tammi, K., Lehmuspelto, T., and Sainio, P. Electric and hybrid electric non-road mobile machinery present situation and future trends. World Electric Vehicle Journal, 2016. 8(1):172-183. doi:10.3390/wevj8010172.

Lauren, M., Goswami, G., Tupitsina, A., Jaiswal, S., Lindh, T., and Sopanen, J. General purpose and scalable internal combustion engine model for energy efficiency studies. Manuscript submitted for publication, -.

Li, L., Zhang, Y., and Yang, C. Model predictive control-based efficient energy recovery control strategy for regenerative braking system of hybrid electric bus. Energy Conversion and Management, 2016. 111:1-15. doi:10.1016/j.enconman.2015.12.077.

Lindgren, M. A transient fuel consumption model for non-road mobile machinery. Biosystems Engineering, 2005. 91(2):139-147. doi:10.1016/j.biosystemseng.2005.03.011.

Lindh, T. Simulation, modeling, and virtual testing of electric-drive-powered mechatronic systems. In 2013 International Conference-Workshop Compatibility And Power Electronics. pages 219-224, 2013. doi:10.1109/CPE.2013.6601158.

Lindh, T., Montonen, J.-H., Niemel, M., Nokka, J., Laurila, L., and Pyrhnen, J. Dynamic performance of mechanical-level hardware-in-the-loop simulation. In 2014 16th European Conference on Power Electronics and Applications. pages 1-10, 2014. doi:10.1109/EPE.2014.6911000.

Lindh, T. and Nevaranta, N. Automatized method of moments to estimate process model of diesel engine dynamics. In 2020 25th IEEE International Conference on Emerging Technologies and Factory Automation (ETFA), volume 1. pages 1201-1204, 2020. doi:10.1109/ETFA46521.2020.9211945.

Markel, T., Brooker, A., Hendricks, T., Johnson, V., Kelly, K., Kramer, B., OKeefe, M., Sprik, S., and Wipke, K. Advisor: a systems analysis tool for advanced vehicle modeling. Journal of Power Sources, 2002. 110(2):255-266. doi:10.1016/S03787753(02)00189-1.

Mocera, F. and Som, A. Analysis of a parallel hybrid electric tractor for agricultural applications. Energies, 2020. 13(12). doi:10.3390/en13123055.

Montonen, J., Montonen, J.-H., Immonen, P., Murashko, K., Ponomarev, P., Tuomo, Lindh, P.,
Laurila, L., and Pyrhnen, J. Electric drive dimensioning for a hybrid working machine by using virtual prototyping. In 2012 XXth International Conference on Electrical Machines. pages 921-927, 2012. doi:10.1109/ICEIMach.2012.6349986.

Montonen, J.-H. and Lindh, T. Analysis of sensorless traction control system for electric vehicle. In 2014 16th European Conference on Power Electronics and Applications. pages 1-7, 2014. doi:10.1109/EPE.2014.6911006.

Moreda, G., Muoz-Garca, M., and Barreiro, P. High voltage electrification of tractor and agricultural machinery a review. Energy Conversion and Management, 2016. 115:117-131. doi:10.1016/j.enconman.2016.02.018.

Nokka, J. Energy Efficiency Analyses of hybrid nonroad mobile machinery by real-time virtual prototyping. Ph.D. thesis, Lappeenranta University of Technology, 2018.

Nokka, J., Laurila, L., and Pyrhnen, J. Virtual simulation-based underground loader hybridization study - comparative fuel consumption and productivity analysis. International Review on Modelling and Simulations (IREMOS), 2017. 10(4). doi:10.15866/iremos.v10i4.12130.

Ottosson, J. Energy Management and Control of Electrical Drives in Hybrid Electrical Vehicles. Ph.D. thesis, Lund University, Sweden, 2007.

Pisu, P. and Rizzoni, G. A comparative study of supervisory control strategies for hybrid electric vehicles. IEEE Transactions on Control Systems Technology, 2007. 15(3):506-518. doi:10.1109/TCST.2007.894649.

Polichronis, D., Evaggelos, R., Alcibiades, G., Gasparakis, E., and Apostolos, P. Turbocharger lubrication - lubricant behavior and factors that cause turbocharger failure. International Journal of Automotive Engineering and Technologies, 2013. 2:40-54.

Rizzoni, G., Guzzella, L., and Baumann, B. Unified modeling of hybrid electric vehicle drivetrains. IEEE/ASME Transactions on Mechatronics, 1999. 4(3):246-257. doi:10.1109/3516.789683.

Rundo, M. Lubrication pumps for internal combustion engines: a review. International Journal of Fluid Power, $2015 . \quad$ 16:59-74. doi:10.1080/14399776.2015.1050935.

Scania. Scania industrial engines dc09 084a. Accessed June. 15, 2021 [Online], -. URL https://www.dlg. org/de/. 
Shabbir, W. and Evangelou, S. A. Exclusive operation strategy for the supervisory control of series hybrid electric vehicles. IEEE Transactions on Control Systems Technology, 2016. 24(6):2190-2198. doi:10.1109/TCST.2016.2520904.

Tammi, K., Minav, T., and Kortelainen, J. Thirty years of electro-hybrid powertrain simulation. IEEE Access, 2018. 6:35250-35259. doi:10.1109/ACCESS.2018.2850916.

Tremblay, O. and Dessaint, L. A. Experimental validation of a battery dynamic model for ev applications. World Electric Vehicle Journal, 2009. 3:289298. doi:10.3390/wevj3020289.
Wang, J., Yang, Z., Liu, S., Zhang, Q., and Han, Y. A comprehensive overview of hybrid construction machinery. Advances in Mechanical Engineering, 2016. 8. doi:10.1177/1687814016636809.

Winke, F. Transient Effects in Simulations of Hybrid Electric Drivetrains. Transient Effects in Simulations of Hybrid Electric Drivetrains. Springer Fachmedien Wiesbaden GmbH, part of Springer Nature, 2019. doi:10.1007/978-3-658-22554-4. 